\title{
A New Perspective on the Average Mixing Matrix
}

\author{
Gabriel Coutinho*, Chris Godsil ${ }^{\dagger} \quad$ Krystal Guo $\$ \$$, Hanmeng Zhan ${ }^{\dagger}$
}

Submitted: May 15, 2018; Accepted: Aug 16, 2018; Published: Oct 19, 2018

(C) The authors. Released under the CC BY-ND license (International 4.0).

\begin{abstract}
We consider the continuous-time quantum walk defined on the adjacency matrix of a graph. At each instant, the walk defines a mixing matrix which is doublystochastic. The average of the mixing matrices contains relevant information about the quantum walk and about the graph. We show that it is the matrix of transformation of the orthogonal projection onto the commutant algebra of the adjacency matrix, restricted to diagonal matrices. Using this formulation of the average mixing matrix, we find connections between its rank and automorphisms of the graph.
\end{abstract}

Mathematics Subject Classifications: 05C88, 05C89

\section{Introduction}

Let $X$ be a graph. We are interested in continuous quantum walks on $X$, which we will define now. The states of the walk are represented by density matrices, positive semidefinite matrices with rows and columns indexed by the vertex set $V(X)$ of $X$, and having trace 1 . If $A$ is the adjacency matrix of $X$, we define the transition matrix $U(t)$ of the walk by

$$
U(t)=\exp (i t A)
$$

The continuous-time quantum walk is an important object of study in quantum computing since it is an universal computational primitive [5]. They were first studied in [7]. Since then, many aspects of quantum walks have been studied, including state transfer $[12,8$, $6,16]$, uniform mixing $[4,10,1]$ and average mixing $[9,2]$. We note that $U(t)$ is unitary and symmetric. If the initial state of the system is given by a density matrix $D$, then the state of the system at time $t$ is

$$
U(t) D U(-t)
$$

\footnotetext{
*Universidade Federal de Minas Gerais, Belo Horizonte, Brazil. gabriel@dcc.ufmg.br

$\dagger$ University of Waterloo, Waterloo, Canada. \{cgodsil, h3zhan\}@uwaterloo.ca

${ }^{\ddagger}$ Université libre de Bruxelles, Brussels, Belgium. guo.krystal@gmail.com

$\S$ This work was done when K. Guo was a postdoctoral fellow at the University of Waterloo.
} 
We note that we do not have direct access to states, all that experiment provides is the value of expressions of the form

$$
\operatorname{tr}\left(D P_{r}\right)
$$

where $P_{1}, \ldots, P_{n}$ are positive semidefinite matrices such that $\sum_{r} P_{r}=I$. Because of this we find ourselves using the trace inner product on $\operatorname{Mat}_{m \times n}(\mathbb{C})$ :

$$
\langle M, N\rangle=\operatorname{tr}\left(M^{*} N\right) .
$$

Unlike classical random walks on a connected graph, a continuous quantum walk does not reach a steady state. Thus, if the eigenvalues of $X$ are integers (e.g., if $X$ is the complete graph $K_{n}$ ) then $U(t)$ is a periodic function of $t$. The focus of this paper, the mixing matrix $\widehat{M}$ of $X$, provides a useful substitute for a steady state.

There are two convenient ways to define $\widehat{M}$. The first is to introduce what we call the mixing matrices $M(t)$ of the walk, given by

$$
M(t)=U(t) \circ \overline{U(t)} .
$$

(Here we use $M \circ N$ to denote the Schur or element-wise product of two matrices of the same order.) Since $U(t)$ is unitary and symmetric, we see that $M(t)$ is symmetric non-negative matrix with each row and column summing to one. We can now define

$$
\widehat{M}=\lim _{T \rightarrow \infty} \frac{1}{T} \int_{0}^{T} M(t) d t .
$$

The study of this time averaging process dates back to von Neumann's Quantum ergodic theorem [17]; for an English translation, see [18]. To see that the first definition makes sense, we recall that since $A$ is real and symmetric, it has a spectral decomposition

$$
A=\sum_{r} \theta_{r} E_{r}
$$

where $\theta_{r}$ runs over the distinct eigenvalues of $A$ and $E_{r}$ is the matrix that represents orthogonal projection onto the $\theta_{r}$-eigenspace of $A$. Given this we also have

$$
U(t)=\sum_{r} e^{i t \theta_{r}} E_{r}
$$

and, as $\overline{U(t)}=U(-t)$,

$$
M(t)=\sum_{r, s} e^{i t\left(\theta_{r}-\theta_{s}\right)} E_{r} \circ E_{s} .
$$

It follows that the limit on the right side of (1) is exists, and

$$
\widehat{M}=\sum_{r} E_{r}^{\circ 2}
$$

that is, the sum of the Schur squares of the spectral idempotents of $A$. This provides an alternative definition of $\widehat{M}$. 
We consider one example. The spectral idempotents of the complete graph $K_{n}$ are

$$
\frac{1}{n} J, \quad I-\frac{1}{n} J,
$$

(with corresponding eigenvalues $n-1$ and -1 ) and hence its average mixing matrix is

$$
\left(1-\frac{2}{n}\right) I+\frac{2}{n^{2}} J
$$

For large $n$, this is very close to $I$. This is unexpected, but we are dealing with quantum physics, where the unexpected is not uncommon.

If $A \in \operatorname{Mat}_{n \times n}(\mathbb{R})$, its commutant $\operatorname{Comm}(A)$ is the set of matrices that commute with $A$. This is an object of some combinatorial interest - the permutation matrices in $\operatorname{Comm}(A)$ are the automorphisms of $A$. The first main result of this paper is that, relative to a natural basis, $\widehat{M}$ is the matrix that represents the restriction to the diagonal matrices of the orthogonal projection from $\operatorname{Mat}_{n \times n}(\mathbb{R})$ onto $\operatorname{Comm}(A)$. We then use this connection to investigate the relations between properties of the graph $X$ and the rank of $\widehat{M}$.

\section{Projection onto the commutant of the adjacency matrix}

Let $A$ be a $n \times n$ real symmetric matrix with columns and rows indexed by elements of a set $V$. We denote by $\operatorname{Mat}_{n \times n}(\mathbb{R})$ the set of $n \times n$ matrices with entries in $\mathbb{R}$. Let the spectral decomposition of $A$ be given as follows:

$$
\sum_{r=1}^{d} \theta_{r} E_{r},
$$

where $\theta_{1}, \ldots, \theta_{m}$ are the distinct eigenvalues of $A$ and $E_{r}$ is the projection matrix onto the $\theta_{r}$ eigenspace of $A$. We denote by $\operatorname{Comm}(A)$ the set of all real matrices which commute with $A$.

Lemma 1. If the eigenvalues $\theta_{1}, \ldots, \theta_{d}$ of $A$ have multiplicities $m_{1}, \ldots, m_{d}$ respectively, them the dimension of $\operatorname{Comm}(A)$ is $\sum_{r} m_{r}^{2}$.

Proof. When $A$ is diagonal, this is immediate. Since $A$ is similar to a diagonal matrix, the lemma follows.

We consider a map $\Psi: \mathbb{R}_{n \times n} \rightarrow \operatorname{Comm}(A)$ such that

$$
\Psi(M)=\sum_{r=1}^{d} E_{r} M E_{r},
$$

for $M \in \mathbb{R}_{n \times n}$. We see that $\Psi$ is an endomorphism of $\mathbb{R}_{n \times n}$. 
Lemma 2. The following are true:

(i) $\Psi$ is idempotent.

(ii) The image of $\Psi$ is equal to $\operatorname{Comm}(A)$.

(iii) $\Psi$ is self-adjoint; that is $\langle M, \Psi(N)\rangle=\langle\Psi(M), N\rangle$.

Proof. Since $E_{r}^{2}=E_{r}$ and $E_{r} E_{s}=0$ if $r \neq s$, it is immediate that $\Psi^{2}(M)=\Psi(M)$ for any matrix $M$. As

$$
A E_{r} M E_{r}=\theta_{r} E_{r} M E_{r}=E_{r} M E_{r} A
$$

we see that $\Psi(M) \in \operatorname{Comm}(A)$ for any $M$. Each idempotent $E_{r}$ is a polynomial in $A$ and consequently if $N \in \operatorname{Comm}(A)$, then

$$
\Psi(N)=\sum_{r} E_{r} N E_{r}=\sum_{r} N E_{r}^{2}=\sum_{r} N E_{r}=N \sum_{r} E_{r}=N I=N .
$$

Hence each element of $\operatorname{Comm}(A)$ lies in the image of $\Psi$ and therefore $\operatorname{im}(\Psi)=\operatorname{Comm}(A)$.

Finally

$$
\left\langle M, E_{r} N E_{r}\right\rangle=\operatorname{tr}\left(M^{T} E_{r} N E_{r}\right)=\operatorname{tr}\left(E_{r} M^{T} E_{r} N\right)=\left\langle E_{r} M E_{r}, N\right\rangle,
$$

from which it follows that $\Psi$ is self-adjoint.

Corollary 3. The map $\Psi$ is the orthogonal projection of the $n \times n$ real matrices onto $\operatorname{Comm}(A)$.

Proof. This is immediate from the fact that $\Psi$ is idempotent and self-adjoint-but we simply note that for any two $n \times n$ matrices $M$ and $N$

$$
\langle\Psi(M), N-\Psi(N)\rangle=\langle M, \Psi(N-\Psi(N))\rangle=\left\langle M, \Psi(N)-\Psi^{2}(N)\right\rangle=0 .
$$

The following is a standard fact in linear algebra, see e.g., [11, Lemma 4.3.1].

Lemma 4. For $B, C, N \in \mathbb{R}_{n \times n}$, we have that

$$
\operatorname{vec}\left(C N B^{T}\right)=(B \otimes C) \operatorname{vec}(N) .
$$

From this we see that, relative to the standard basis of $\operatorname{Mat}_{n \times n}(\mathbb{R})$, the matrix that represents $\Psi$ is $\sum_{r} E_{r} \otimes E_{r}$. (Since $\operatorname{tr}\left(E_{r}\right)=m_{r}$ it follows that $\operatorname{tr}(\Psi)=\sum_{r} m_{r}^{2}$; since $P$ is idempotent $\operatorname{tr}(P)=\operatorname{rk}(P)$ and thus we have a second proof of Lemma 1.) 


\section{Average states}

Recall that a state $D$ is a positive semidefinite matrix with trace 1 . If $D$ is the initial state of a continuous quantum walk, then the state $D(t)$ at time $t$ is

$$
D(t)=U(t) D U(-t) .
$$

Using the spectral decomposition of $U(t)$, we have

$$
D(t)=\sum_{r, s} e^{i t\left(\theta_{r}-\theta_{s}\right)} E_{r} D E_{s}
$$

whence

$$
\lim _{T \rightarrow \infty} \frac{1}{T} \int_{0}^{T} D(t) d t=\sum_{r} E_{r} D E_{r} .
$$

We call this limit (or sum) an average state and denote it by $\Psi(D)$. We see at once that $\Psi(D)$ is equal to the orthogonal projection of $D$ onto $\operatorname{Comm}(A)$.

Since $D$ is positive semidefinite and the idempotents $E_{r}$ are symmetric, we see that $\Psi(D)$ is a positive semidefinite matrix. As

$$
\operatorname{tr}(\Psi(D))=\sum_{r} \operatorname{tr}\left(E_{r} D E_{r}\right)=\sum_{r} \operatorname{tr}\left(D E_{r}^{2}\right)=\sum_{r} \operatorname{tr}\left(D E_{r}\right)=\operatorname{tr}(D)
$$

we also see that $\Psi(D)$ is a density matrix. We remark that the map

$$
D \mapsto \sum_{r} E_{r} D E_{r}
$$

is a quantum channel; for more background on quantum channels, see [3, 13, 14].

In the context of quantum walks on graphs, there is a natural class of density matrices we will focus on. If $a \in V(X)$, let $e_{a}$ denote the standard basis vector of $\mathbb{C}^{V(X)}$ indexed by $a$ and define $D_{a}=e_{a} e_{a}^{T}$. Then certainly $D_{a}$ is a density matrix, moreover $\operatorname{rk}\left(D_{a}\right)=1$ and $D_{a}^{2}=D_{a}$. (Physicists refer to a density matrix with rank 1 as a pure state.)

The following theorem gives one reason why average states are of interest. Given a set of vectors $v_{1}, \ldots, v_{n}$ in an inner product space, the Gram matrix of $\left\{v_{1}, \ldots, v_{n}\right\}$ is the $n \times n$ matrix $G$ with $j k$-entry

$$
G_{j k}:=\left\langle v_{j}, v_{k}\right\rangle .
$$

Theorem 5. Let $X$ be a graph. The average mixing matrix is the Gram matrix of the average states $\Psi\left(D_{a}\right)$ for a in $V(X)$.

Proof. Our claim is that, if $a, b \in V(X)$, then

$$
\left\langle\Psi\left(D_{a}\right), \Psi\left(D_{b}\right)\right\rangle=(\widehat{M})_{a, b} .
$$

Now

$$
\Psi\left(D_{a}\right) \Psi\left(D_{b}\right)=\sum_{r} E_{r} D_{a} E_{r} \sum_{s} E_{s} D_{b} E_{s}
$$


and so

$$
\operatorname{tr}\left(\Psi\left(D_{a}\right) \Psi\left(D_{b}\right)\right)=\sum_{r} \operatorname{tr}\left(E_{r} D_{a} E_{r} D_{b} E_{r}\right)
$$

Further

$$
E_{r} D_{a} E_{r} D_{b} E_{r}=E_{r} e_{a} e_{a}^{T} E_{r} e_{b} e_{b}^{T} E_{r}=\left(E_{r}\right)_{a, b} E_{r} e_{a} e_{b}^{T} E_{r}
$$

and therefore

$$
\operatorname{tr}\left(\Psi\left(D_{a}\right) \Psi\left(D_{b}\right)\right)=\sum_{r}\left(E_{r}\right)_{a, b}\left(E_{r}\right)_{b, a}=\sum_{r}\left(E_{r}^{\circ 2}\right)_{a, b}
$$

and the theorem follows.

Corollary 6. The dimension of the space spanned by the average state $\Psi\left(D_{a}\right)$ for a $\in$ $V(X)$ is equal to $\operatorname{rk}(\widehat{M})$.

Note that a matrix which can be written as the Gram matrix of a set of vectors is positive semidefinite. If these vectors are themselves positive semidefinite matrices, then the resulting Gram matrix is called completely positive semidefinite. This concept has been introduced quite recently and was investigated in the context of conic optimization for certain quantum parameters of graphs, so we still do not know exactly what to do with the information. Nevertheless, we state the corollary below.

Corollary 7. The average mixing matrix is completely positive semidefinite.

\section{Diagonal matrices in the commutant}

In this section, we consider the restriction of $\Psi$ to the set of $n \times n$ diagonal matrices. Let $\mathcal{D}$ be the set of $n \times n$ diagonal matrices. The standard basis of $\mathcal{D}$ is given by $\left\{D_{a}\right\}_{a \in V}$ where $D_{a}:=e_{a} e_{a}^{T}$.

The map that sends a matrix $M$ to $\Psi(M) \circ I$ is a linear map from $\operatorname{Mat}_{n \times n}(\mathbb{R})$ into the space $\mathcal{D}$ of diagonal matrices. We denote the restriction of this map to $\mathcal{D}$ by $\Phi$; it is evidently an endomorphism of $\mathcal{D}$.

Lemma 8. Relative to the standard basis $\left\{D_{a}\right\}_{a \in V}$ of $\mathcal{D}$, the matrix that represents $\Phi$ is $\widehat{M}$. Hence $\operatorname{rk}(\widehat{M})=\operatorname{dim}(\Phi(\mathcal{D}))$.

Proof. The entries of the matrix representing $\Psi$ are given by the the inner products $\left\langle D_{a}, \Psi\left(D_{b}\right)\right\rangle$ for vertices $a$ and $b$ of $X$. We have

$$
\left\langle D_{a}, \Psi\left(D_{b}\right)\right\rangle=\sum_{r} \operatorname{tr}\left(D_{a} E_{r} D_{b} E_{r}\right)
$$

and

$$
\operatorname{tr}\left(D_{a} E_{r} D_{b} E_{r}\right)=\operatorname{tr}\left(e_{a} e_{a}^{T} E_{r} e_{b} e_{b}^{T} E_{r}\right)=\left(E_{r}\right)_{a, b}\left(E_{r}\right)_{b, a}=\left(E_{r}^{\circ 2}\right)_{a, b}
$$

Hence

$$
\left\langle D_{a}, \Psi\left(D_{b}\right)\right\rangle=\widehat{M}_{a, b}
$$


Lemma 9. If $D$ is diagonal, then $\Psi(D)=0$ if and only if $I \circ \Psi(D)=0$.

Proof. Since $\operatorname{ker}(\Psi)=\operatorname{Comm}(A)^{\perp}$, we see that $\Psi(D)=0$ if and only if $D \in \operatorname{Comm}(A)^{\perp}$. Note that if $D$ is diagonal, then

$$
\langle D, N\rangle=\langle D, I \circ N\rangle
$$

Assume $D$ is diagonal and $I \circ \Psi(D)=0$. Then for each vertex $a$ of $X$, we have

$$
0=\left\langle D_{a}, I \circ \Psi(D)\right\rangle=\left\langle D_{a}, \Psi(D)\right\rangle=\left\langle\Psi\left(D_{a}\right), \Psi(D)\right\rangle
$$

and so $\Psi(D)$ is orthogonal to each matrix $\Psi\left(D_{a}\right)$. Since $\Psi(D)$ lies in the span of the matrices $\Psi\left(D_{a}\right)$, we conclude that $\Psi(D)=0$.

Let $\mathcal{A}_{0}$ denote the set of matrices in $\operatorname{Comm}(A)$ with all diagonal entries equal to 0; that is

$$
\mathcal{A}_{0}=\{N \in \operatorname{Comm}(A): N \circ I=0\} .
$$

Observe that $\mathcal{A}_{0}$ is always non-empty, since $A \in \mathcal{A}_{0}$. Also $I \in \Psi(\mathcal{D})$, and therefore the direct sum decomposition in our next result is always non-trivial.

Lemma 10. $\operatorname{Comm}(A)=\Psi(\mathcal{D}) \oplus \mathcal{A}_{0}$

Proof. If $M \in \mathcal{A}_{0}$, then $\Psi(M)=M$ and so

$$
\left\langle\Psi\left(D_{a}\right), M\right\rangle=\left\langle D_{a}, \Psi(M)\right\rangle=\left\langle D_{a}, M\right\rangle=\operatorname{tr}\left(e_{a} e_{a}^{T} M\right)=M_{a, a} .
$$

Accordingly $M$ is orthogonal to each matrix $\Psi\left(D_{a}\right)$ for $a$ in $V(X)$ if and only if $M \circ I=$ 0 .

\section{The rank of some average mixing matrices}

There are a number of graph invariants that can be constructed from the average mixing matrix. In this section we focus on $\operatorname{rk}(\widehat{M})$. From $[9]$ we know that if $\operatorname{rk}(\widehat{M})=1$, then $X$ is $K_{1}$ or $K_{2}$.

Our first two results concern graphs with only simple eigenvalues; we note that almost all graphs have this property [15].

Lemma 11. Assume $X$ is a graph with simple eigenvalues on $n$ vertices. If $n \geqslant 2$, then $\operatorname{rk}(\widehat{M}) \leqslant n-1$. Further, if $X$ is regular and $n \geqslant 4$, then $\operatorname{rk}(\widehat{M}) \leqslant n-3$.

Proof. Since the eigenvalues of $X$ are simple, $\operatorname{dim}(\operatorname{Comm}(A))=n$ and since $A \in \mathcal{A}_{0}$, it follows from Lemma 10 that $\operatorname{dim}(\Psi(\mathcal{D})) \leqslant n-1$.

Suppose $X$ is regular with valency $k$. Then the matrices $A, J-I$ and $A^{2}-k I$ all lie in $\mathcal{A}_{0}$. If these matrices are linearly dependent, the minimal polynomial of $A$ has degree at most two and hence $A$ has at most two eigenvalues. Since $X$ has $n$ distinct eigenvalues, the minimal polynomial of $A$ over $\mathbf{1}^{\perp}$ has degree $n-1$. Thus $n-1 \leqslant 2$, which contradicts our assumption that $n \geqslant 4$. 
Table 1 shows the number of graphs on $n$ vertices whose mixing matrices have given ranks. The only regular graph with simple eigenvalues on up to 8 vertices is $K_{2}$, so the data for regular graphs is not included in this table. Instead, we count cubic graphs with simple eigenvalues on 10 to 18 vertices, as presented in Table 2.

Lemma 12. Assume $X$ is a graph on $n$ vertices with only simple eigenvalues. If $X$ is bipartite, then $\operatorname{rk}(\widehat{M}) \leqslant\lfloor(n+1) / 2\rfloor$.

Proof. Note that $A^{k} \in \mathcal{A}_{0}$ if and only if $k$ is odd, and for all $k<n$, these matrices are independent. Hence $\operatorname{dim} \mathcal{A}_{0} \geqslant\lfloor n / 2\rfloor$, and the result follows from Lemmas 8 and 10 .

\begin{tabular}{|c|c|c|c|}
\hline$n$ & $\operatorname{rk}(\widehat{M})$ & \# graphs & \# simple eigenvalues \\
\hline$\overline{3}$ & 2 & 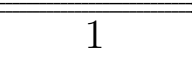 & 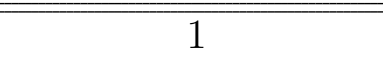 \\
\hline 3 & 3 & 1 & 0 \\
\hline 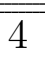 & 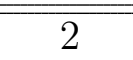 & 3 & $\overline{2}$ \\
\hline 4 & 3 & 1 & 1 \\
\hline 4 & 4 & 2 & 0 \\
\hline 5 & 3 & 11 & 8 \\
\hline 5 & 4 & 6 & 3 \\
\hline 5 & 5 & 4 & 0 \\
\hline 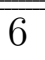 & $\overline{2}$ & $\overline{2}$ & $\overline{2}$ \\
\hline 6 & 3 & 27 & 12 \\
\hline 6 & 4 & 32 & 21 \\
\hline 6 & 5 & 35 & 19 \\
\hline 6 & 6 & 16 & 0 \\
\hline 7 & 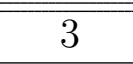 &  & $\overline{5}$ \\
\hline 7 & 4 & 189 & 121 \\
\hline 7 & 5 & 240 & 158 \\
\hline 7 & 6 & 352 & 255 \\
\hline 7 & 7 & 66 & 0 \\
\hline 8 & $\overline{2}$ & $\overline{3}$ & $\overline{3}$ \\
\hline 8 & 3 & 39 & 25 \\
\hline 8 & 4 & 466 & 236 \\
\hline 8 & 5 & 1360 & 776 \\
\hline 8 & 6 & 2523 & 1492 \\
\hline 8 & 7 & 5781 & 4787 \\
\hline 8 & 8 & 945 & 0 \\
\hline
\end{tabular}

Table 1: Number of graphs with given $\operatorname{rk}(\widehat{M})$. 


\begin{tabular}{|c|c|c|c|}
\hline$n$ & $\operatorname{rk}(\widehat{M})$ & $\begin{array}{l}\text { \# cubic } \\
\text { graphs }\end{array}$ & $\begin{array}{c}\text { \# simple } \\
\text { eigenvalues }\end{array}$ \\
\hline 10 & 3 & 2 & 2 \\
\hline 10 & 5 & 8 & 1 \\
\hline 10 & 6 & 5 & 3 \\
\hline 10 & 7 & 1 & 0 \\
\hline 10 & 10 & 3 & 0 \\
\hline$\overline{12}$ & $\overline{3}$ & $\overline{\overline{1}}$ & 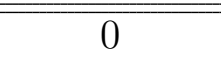 \\
\hline 12 & 4 & 3 & 0 \\
\hline 12 & 5 & 8 & 3 \\
\hline 12 & 6 & 11 & 2 \\
\hline 12 & 7 & 18 & 6 \\
\hline 12 & 8 & 14 & 4 \\
\hline 12 & 9 & 14 & 3 \\
\hline 12 & 10 & 11 & 0 \\
\hline 12 & 11 & 2 & 0 \\
\hline 12 & 12 & 3 & 0 \\
\hline$\overline{14}$ & 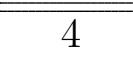 & $\overline{13}$ & $\overline{12}$ \\
\hline 14 & 5 & 19 & 12 \\
\hline 14 & 6 & 30 & 7 \\
\hline 14 & 7 & 82 & 37 \\
\hline 14 & 8 & 97 & 65 \\
\hline 14 & 9 & 66 & 37 \\
\hline 14 & 10 & 62 & 45 \\
\hline 14 & 11 & 117 & 101 \\
\hline 14 & 12 & 18 & 0 \\
\hline 14 & 13 & 3 & 0 \\
\hline 14 & 14 & 2 & 0 \\
\hline
\end{tabular}

\begin{tabular}{|c|c|c|c|}
\hline$n$ & $\operatorname{rk}(\widehat{M})$ & $\begin{array}{c}\text { \# cubic } \\
\text { graphs }\end{array}$ & $\begin{array}{l}\text { \# simple } \\
\text { eigenvalues }\end{array}$ \\
\hline 16 & 4 & 4 & 3 \\
\hline 16 & 5 & 45 & 29 \\
\hline 16 & 6 & 58 & 11 \\
\hline 16 & 7 & 122 & 49 \\
\hline 16 & 8 & 252 & 112 \\
\hline 16 & 9 & 393 & 220 \\
\hline 16 & 10 & 359 & 144 \\
\hline 16 & 11 & 311 & 141 \\
\hline 16 & 12 & 684 & 464 \\
\hline 16 & 13 & 1365 & 1008 \\
\hline 16 & 14 & 366 & 0 \\
\hline 16 & 15 & 77 & 0 \\
\hline 16 & 16 & 24 & 0 \\
\hline$\overline{18}$ & $\overline{5}$ & $\overline{48}$ & $\overline{45}$ \\
\hline 18 & 6 & 147 & 59 \\
\hline 18 & 7 & 226 & 78 \\
\hline 18 & 8 & 414 & 152 \\
\hline 18 & 9 & 1268 & 724 \\
\hline 18 & 10 & 1785 & 982 \\
\hline 18 & 11 & 1865 & 842 \\
\hline 18 & 12 & 1264 & 539 \\
\hline 18 & 13 & 1940 & 1146 \\
\hline 18 & 14 & 7254 & 5819 \\
\hline 18 & 15 & 19302 & 16060 \\
\hline 18 & 16 & 4763 & 0 \\
\hline 18 & 17 & 643 & 0 \\
\hline 18 & 18 & 382 & 0 \\
\hline
\end{tabular}

Table 2: Number of cubic graphs with given $\operatorname{rk}(\widehat{M})$.

Lemma 13. Let $S$ be a proper subset of the vertices of the graph $X$, and assume that for each vertex a in $S$, there is an automorphism of $X$ with a as its only fixed point. Then $\operatorname{rk}(\widehat{M}) \geqslant|S|+1$.

Proof. We identify the automorphism group of $X$ with the set of permutation matrices that lie in $\operatorname{Comm}(A)$. If $a \in S$, let $P_{a}$ be the automorphism of $X$ with $a$ as its only fixed point. From Lemma 10, for each $P_{a}$, there is a matrix in $\Psi(\mathcal{D})$ with the same diagonal. These matrices and the identity matrix form a set of linearly independent matrices in $\Psi(\mathcal{D})$. 
Table 3 shows the number of bipartite graphs on $n$ vertices whose mixing matrices have given ranks.

\begin{tabular}{|c|c|c|c|}
\hline$n$ & $\operatorname{rk}(\widehat{M})$ & \# bipartite graphs & \# simple eigenvalues \\
\hline \hline 3 & 2 & 1 & 1 \\
\hline 3 & 3 & 0 & 0 \\
\hline \hline 4 & 2 & 2 & 1 \\
\hline 4 & 3 & 0 & 0 \\
\hline 4 & 4 & 1 & 0 \\
\hline \hline 5 & 3 & 3 & 3 \\
\hline 5 & 4 & 1 & 0 \\
\hline 5 & 5 & 1 & 0 \\
\hline \hline 6 & 2 & 1 & 1 \\
\hline 6 & 3 & 6 & 3 \\
\hline 6 & 4 & 4 & 0 \\
\hline 6 & 5 & 4 & 0 \\
\hline 6 & 6 & 2 & 0 \\
\hline \hline 7 & 3 & 0 & 0 \\
\hline 7 & 4 & 23 & 20 \\
\hline 7 & 5 & 3 & 0 \\
\hline 7 & 6 & 1 & 0 \\
\hline 7 & 7 & 3 & 0 \\
\hline \hline 8 & 2 & 1 & 1 \\
\hline 8 & 3 & 5 & 2 \\
\hline 8 & 4 & 43 & 0 \\
\hline 8 & 5 & 51 & 0 \\
\hline 8 & 6 & 50 & 0 \\
\hline 8 & 7 & 21 & 0 \\
\hline 8 & 8 & 11 & 0 \\
\hline & & & 0 \\
\hline
\end{tabular}

Table 3: Number of bipartite graphs with given $\operatorname{rk}(\widehat{M})$.

If $X$ is a Cayley graph for an abelian group of odd order, then since the map that sends each group element to its inverse gives rise to an automorphism of the Cayley graph with 1 as its only fixed point, it follows that each vertex is the unique fixed point of an automorphism of $X$. This implies $\widehat{M}$ must be invertible in this case. One easy consequence is that the average mixing matrix of a cycle is invertible (although this is also an a consequence of results in [9]).

Some of our numerical data indicates that for most graphs on $n$ vertices with simple eigenvalues, the average mixing matrix has rank $n-1$. In view of Theorem 10 , this implies that, for these graphs, $\operatorname{dim} \mathcal{A}_{0}=1$. Therefore any matrix that commutes with $A$ and has zero diagonal must be a scalar multiple of $A$. The following is an immediate consequence. 
Corollary 14. Suppose $X$ is a connected graph with at least three vertices. If all eigenvalues of $X$ are simple and $\operatorname{rk}(\widehat{M})=n-1$, then any automorphism of $X$ has fixed points.

\section{Open problems}

As the theory of the average mixing matrix is relatively new, there are many interesting problems one can ask. We discuss a few below.

One interesting question about the average mixing matrix concerns the non-negative rank of the average mixing matrix. The non-negative rank of a non-negative $n \times n$ matrix $A$ is the least number $k$, such that there are $k$ matrices $\left\{M_{r}\right\}_{r=1}^{k}$ of rank 1 with nonnegative entries, such that $A=\sum_{r=1}^{k} M_{r}$. If $X$ is a graph on $n>1$ vertices with simple eigenvalues, Lemma 11 gives that $\widehat{M}$ has rank at most $n-1$. One can ask when the non-negative rank of $\widehat{M}$ is equal to $n$.

We may interpret the $(v, v)$ diagonal entry of $\widehat{M}$ as the average probability of measuring at vertex $v$, after starting at vertex $v$. Thus questions about the trace of $\widehat{M}$ are very natural. In particular, it is interesting to ask how graph invariants correspond to the trace of $\widehat{M}$. Following in the vein of the questions about rank and trace, one can ask if the spectrum of $\widehat{M}$ determines any graph properties. This gives rise to many natural questions about the average mixing matrix.

\section{Acknowledgements}

GC acknowledges the support of grants FAPESP 15/16339-2 and CNPq 459335/2014-6. CG acknowledges the support of NSERC Grant RGPIN-9439.

\section{References}

[1] William Adamczak, Kevin Andrew, Leon Bergen, Dillon Ethier, Peter Hernberg, Jennifer Lin, and Christino Tamon, Non-Uniform Mixing Of Quantum Walk On Cycles, International Journal Of Quantum Information 05 (2007), no. 06, 781-793.

[2] William Adamczak, Kevin Andrew, Peter Hernberg, and Christino Tamon, A note on graphs resistant to quantum uniform mixing, arXiv:quant-ph/0308073 (2003), 9 .

[3] Cédric Bény and Florian Richter, Algebraic approach to quantum theory: a finitedimensional guide, arXiv:1505.03106 (2018).

[4] Ada Chan, Complex Hadamard Matrices, Instantaneous Uniform Mixing and Cubes, ArXiv e-prints (2013).

[5] Andrew M Childs, Universal computation by quantum walk, Physical Review Letters 102 (2009), no. 18, 4,180501. 
[6] G. Coutinho, C. Godsil, K. Guo, and F. Vanhove, Perfect state transfer on distanceregular graphs and association schemes, Linear Algebra and its Applications 478 (2015), $108-130$.

[7] Edward Farhi and Sam Gutmann, Quantum computation and decision trees, Physical Review A 58 (1998), no. 2, 915-928.

[8] Chris D Godsil, State transfer on graphs, Discrete Mathematics 312 (2012), no. 1, 129-147.

[9] _ Average mixing of continuous quantum walks, Journal of Combinatorial Theory, Series A 120 (2013), no. 7, 1649-1662.

[10] Chris D Godsil, Natalie Mullin, and Aidan Roy, Uniform Mixing and Association Schemes, Electron. J. Combin. 24 (2013), no. 3, \#P3.22.

[11] Roger A Horn and Charles R Johnson, Topics in matrix analysis, Cambridge university press, 1991.

[12] Alastair Kay, Perfect, efficient, state transfer and its application as a constructive tool, International Journal of Quantum Information 8 (2010), no. 04, 641-676.

[13] Phillip Kaye, Raymond Laflamme, and Michele Mosca, An introduction to quantum computing, Oxford University Press, 2007.

[14] Michael Nielsen and Isaac Chuang, Quantum Computation and Quantum Information, Cambridge University Press, Cambridge, 2010.

[15] Terence Tao and Van $\mathrm{Vu}$, Random matrices have simple spectrum, Combinatorica 37 (2017), no. 3, 539-553.

[16] Luc Vinet and Alexei Zhedanov, How to construct spin chains with perfect state transfer, Physical Review A 85 (2012), no. 1, 12323.

[17] John von Neumann, Beweis des Ergodensatzes und des H-Theorems, Zeitschrift fuer Physik (1929), 30-70.

[18] _ Proof of the ergodic theorem and the H-theorem in quantum mechanics, Eur. Phys. J. H 35 (2010), 201 - 237. (Translated by Roderich Tumulka). 